\title{
Magnetic Viscosity and Magnetization Reversal of Acicular Barium Ferrite Particles
}

\author{
H. NISHIO ${ }^{1}$, K. KAKIZAKI ${ }^{2}$, and N. HIRATSUKA ${ }^{2}$
}

1) Materials Research Center, TDK Corporation, Minami-Hatori, Narita 286, Japan

2) Faculty of Engineering, Saitama University, 255 Simo-Okubo, Urawa 338, Japan

\begin{abstract}
The magnetic viscosity coefficient (Sv), the activation volume (Vact), the rotational hysteresis integral (Rh) and the average anisotropy field $\left(\mathrm{H}_{\mathrm{A}}\right.$, ave) were examined for acicular $\mathrm{BaCo}_{\mathrm{x}} \mathrm{Ti}_{\mathrm{x}} \mathrm{Fe}_{12-2 \mathrm{x}} \mathrm{O}_{19}(\mathrm{x}=0-1.0)$ particles prepared by a conventional sintering method. Acicular hexagonal Ba-ferrite particles with different coercive force $(\mathrm{Hcj}=35-310 \mathrm{MA} / \mathrm{m}$ ) were prepared, and the dependence of Vact, $\mathrm{Rh}$ and $\mathrm{H}_{\mathrm{A}}$, ave on Hcj was investigated. The results show that Hoj is proportional to Vact ${ }^{-2.2}$, as in the case of the platelet Ba-ferrite particles, SrM particles, $\mathrm{SrW}$ and $\mathrm{Sm}_{2} \mathrm{Fe}_{17} \mathrm{~N}_{x}$ magnets, which all have a similar mechanism of magnetization reversal. Also, $\mathrm{H}_{\mathrm{A}}$, ave increases with increasing $\mathrm{Hcj}$ and Vact is inversely proportion to the average anisotropy constant. It was found that $\mathrm{Rh}$ and Vact both decrease, as $\mathrm{Hcj}$ increases, to approach the coherent rotation mode.
\end{abstract}

\section{I.INTRODUCTION}

Many report on magnetization reversal of hexagonal platelet Ba-ferrite particles have been published [14]. Recently, there has been much interest in acicular hexagonal $\mathrm{Ba}$-ferrite particles prepared by a conventional sintering method [5], though studies on their magnetic viscosity and on the mechanism of magnetization reversal are few [6]. The magnetic viscosity in fine pariicles used for recording media belongs to the Jordan-type aftereffect and is caused by thermal fluctuations [4,7]. It is known to depend on the magnetic viscosity coefficient (Sv), the irreversible susceptibility (Xirr) and the logarithm of time $(\ln t)$ [8-10]. When the change in magnetization $(\Delta d)$ from time $t_{1}$ to $t_{2}$ is considered, Sv can be expressed as $: S v=\Delta J / X i r r\left(\ln t_{1} \cdot \ln t_{2}\right)=k T /$ (Vact Js), where $\mathrm{k}$ is Boltzmann constant, Vact is the activation volume and $\mathrm{Js}$ is the saturation magnetization [8-10].

This paper reports on the dependence of Sv, Vact, the rotational hysteresis integral $(\mathrm{Rh})$ and the average anisotropy field $\left(\mathrm{H}_{\mathrm{A}}\right.$, ave) on coercive force (Hcj) for acicular Ba-ferrite particles prepared by a conventional sintering method.

\section{II .EXPERIMENTAL}

Acicular $\alpha$ - $\mathrm{FeOOH}, \mathrm{Ba}(\mathrm{OH})_{2} \cdot 8 \mathrm{H}_{2} \mathrm{O}, \mathrm{C}_{10} \mathrm{H}_{14} \mathrm{O}_{4} \mathrm{Co}$ $2 \mathrm{H}_{2} \mathrm{O}$ and $\mathrm{C}_{10} \mathrm{H}_{14} \mathrm{O}_{4}$ TiO were previously weighed for the formation of $\mathrm{BaCo}_{\mathrm{x}} \mathrm{Ti}_{\mathrm{x}} \mathrm{Fe}_{12-2 \mathrm{x}} \mathrm{O}_{19}$ with the doping rate $\mathrm{X}$ ranging from 0 to 1.0 [5]. The starting material for the acicular $\alpha \cdot \mathrm{FeOOH}$ particles had a long axis of about $1.3 \mu \mathrm{m}$ and an aspect ratio of about 33 . The $\mathrm{Ba}(\mathrm{OH})_{2} \cdot 8 \mathrm{H}_{2} \mathrm{O}$ was dissolved in warm water and the acicular $\alpha \cdot \mathrm{FeOOH}$ particles, $\mathrm{C}_{10} \mathrm{H}_{14} \mathrm{O}_{4} \mathrm{Co} \cdot 2 \mathrm{H}_{2} \mathrm{O}$ and $\mathrm{C}_{10} \mathrm{H}_{14} \mathrm{O}_{4}$ TiO were added. The liquid mixture was coated on a stainless steel plate which was heated to about $150^{\circ} \mathrm{C}$ to be dried. The dried mixtures were sintered at $900^{\circ} \mathrm{C}$ for $3 \mathrm{~h}$ in air and then quenched.

Table 1 shows the magnetic properties measured using a vibrating sample magnetometer (VSM) for the acicular $\mathrm{BaCo}_{x} \mathrm{Ti}_{x} \mathrm{Fe}_{12 \cdot 2 \mathrm{x}} \mathrm{O}_{19}$ particles so produced, with $\mathrm{X}$ ranging from 0 to 1.0 . These particles consisted of hexagonal grains (6-10 in number), whose easy axis was in the c-direction, perpendicular to that in acicular particles (a-direction) [6]. The mean diameter and length of the particles (obtained by a TEM) were $0.07 \times 0.8 \mu \mathrm{m}$ respectively (the mean particle volume, $V \sim 30 \times 10^{-22} \mathrm{~m}^{3}$ ).

After the application of $1.6 \mathrm{MA} / \mathrm{m}$, the samples were subjected to a constant magnetic field $(\mathrm{H})$ for $1000 \mathrm{~s}$ and $\Delta \mathrm{J}$ measured using a VSM [7]. The reversible susceptibility (Xrev) and the total differential susceptibility (Xtot) were also measured using a VSM.

Table 1. Magnetic properties of acicular BaCoxTix Fe12-2xO19 particles with $X$ ranging from 0 to 1.0.

\begin{tabular}{rcc}
\hline $\mathrm{X}$ & $\mathrm{J} \mathbf{s}\left(\times 10^{-6} \mathrm{~Wb} \mathrm{~m} / \mathrm{kg}\right)$ & $\mathrm{Hoj}(\mathrm{kA} / \mathrm{m})$ \\
\hline 0 & 45.2 & 308 \\
0.5 & 43.5 & 137 \\
0.7 & 42.7 & 86 \\
1.0 & 39.7 & 34 \\
\hline
\end{tabular}


Xirr can be expressed as Xtot-Xrev.

The rotational hysteresis loss $(\mathrm{Wr})$ was measured using a torque magnetometer. Rh, which corresponds to the mechanism of magnetization reversal, is given by the equation $R h=\int \mathrm{Wr} / \mathrm{Js} d(1 / \mathrm{H})[11] . H_{\mathrm{A}}$, ave was calculated from the remanent magnetization (Ji) which was measured using a previous described method employing a VSM at increment of $40 \mathrm{kA} / \mathrm{m}$ for angles between $0^{\circ}$ and $5^{\circ} \quad[4,12]$. The average anisotropy constant $\left(\mathrm{K}_{\mathrm{A}}\right.$, ave $)$ is calculated from the equation $\mathrm{K}_{\mathrm{A}}$, ave $=1 / 2 \mathrm{H}_{\mathrm{A}}$, ave $\times$ Js.

\section{III . RESULTS AND DISCUSSION}

Fig. 1 shows the magnetic field dependence of $\mathrm{Sv}$ obtained from the values of $\Delta J /\left(\ln t_{1}-\ln t_{2}\right)$ and Xirr. The value of Sv, obtained around Hcj point for all samples, is almost independent of the magnetic field. In this experiment, the logarithm of Sv varies in proportion to $\ln \mathrm{Hcj}$ as given in the generally accepted Barbier's relationship [7,13].

The author had reported that the relation of $\mathrm{Hcj} \propto$ Vact ${ }^{0.67}$ was obtained for acicular $\gamma \cdot \mathrm{Fe}_{2} \mathrm{O}_{3}$, Co $\gamma$ $\mathrm{Fe}_{2} \mathrm{O}_{3}$ and metal particles used for recording media [7]. For acicular Ba-ferrite particles, Hcj is proportional to Vact ${ }^{2.2}$ as shown in Fig. 2, nearly the same as in the case of the platelet Ba-ferrite particles $\left(\mathrm{Hcj} \propto\right.$ Vact $\left.^{-1.9}\right)$ used for recording media [7], SrM particles $\left(\mathrm{Hcj} \propto\right.$ Vact $\left.^{2.0}\right)$ used in permanent magnets [14], SrW magnets (Hcj $\propto$ Vact ${ }^{-2.1}$ ) [15] and $\mathrm{Sm}_{2} \mathrm{Fe}_{17} \mathrm{~N}_{\mathrm{x}}$ compacted powder magnets $\left(\mathrm{Hcj} \propto \mathrm{Vact}^{-2.0}\right)$ [16]. It is thus believed that they all have a similar mechanism of magnetization reversal. In the case of the platelet Ba-ferrite particles, V/Vact was approximately 1.4 to 2.3 at $293 \mathrm{~K}$ [4]. However, for acicular Ba-ferrite particles, V/Vact is approximately 90 .

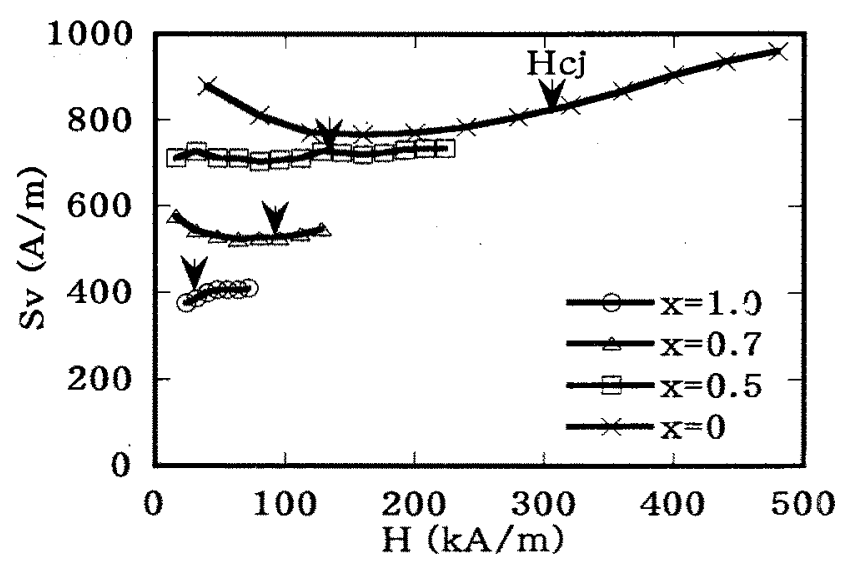

Fig. 1 Magnetic field dependence of the magnetic viscosity coefficient $\mathrm{Sv}$ for acicular $\mathrm{BaCo}_{x} \mathrm{Ti}_{x} \mathrm{Fe}_{12-2 x} \mathrm{O}_{19}$ particles.

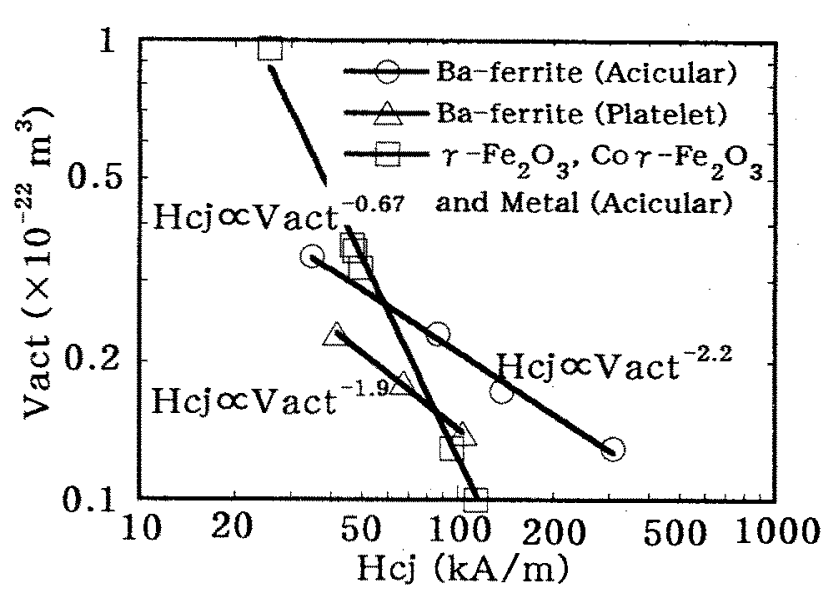

Fig. 2 Plots of the activation volume Vact against coercive force Hcj for acicular and platelet Ba-ferrite, acicular $\boldsymbol{\gamma}$ $\mathrm{Fe}_{2} \mathrm{O}_{3}$, Co $\gamma-\mathrm{Fe}_{2} \mathrm{O}_{3}$ and metal particles.

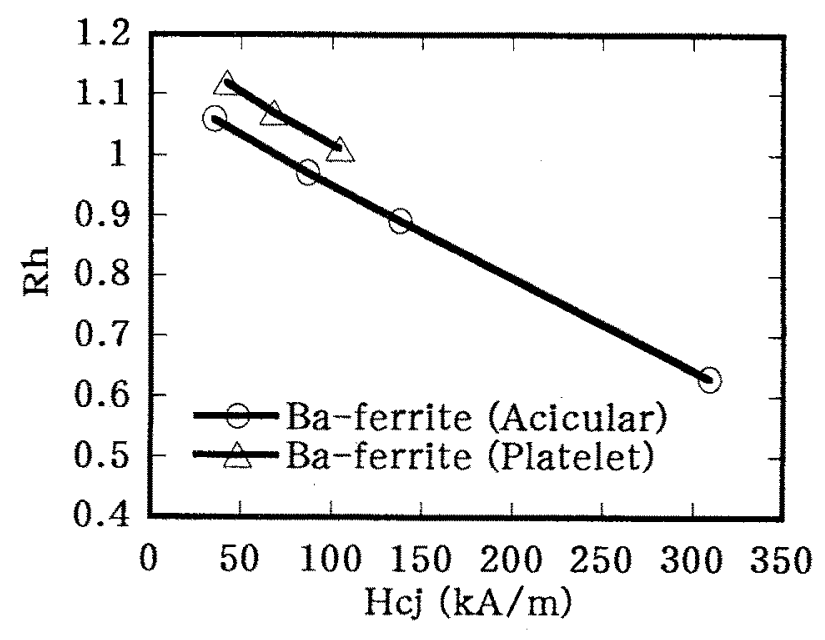

Fig. 3 Rotational hysteresis integral Rh against coercive force $\mathrm{Hcj}$ for acicular and platelet $\mathrm{Ba}$-ferrite particles.

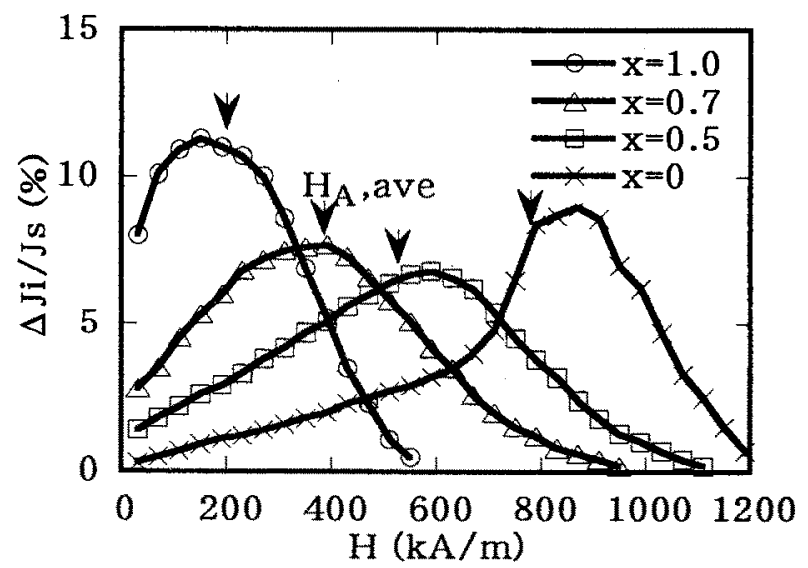

Fig. 4 Anisotropy field $\mathrm{H}_{\mathrm{A}}$ distribution of acicular $\mathrm{BaCo}_{\mathrm{x}} \mathrm{Ti}_{\mathrm{x}} \mathrm{Fe}_{12-2 \mathrm{x}} \mathrm{O}_{19}$ particles. 
Fig. 3 plots the relationship between Hcj and Rh calculated using the $\mathrm{Wr} / \mathrm{Js}$ versus $1 / \mathrm{H}$ curves for the acicular and platelet Ba-ferrite particles. The Rh value decreases as Hcj increases. This suggests that the mechanism of magnetization reversal approaches coherent rotation, namely, the coherency increases as $\mathrm{Hcj}$ increases. The $\mathrm{Rh}$ value for $\mathrm{X}=0\left(\mathrm{BaFe}_{12} \mathrm{O}_{19}\right)$ is closer to the value calculated from the coherent rotation mode $(\mathrm{Rh}=0.4)$.

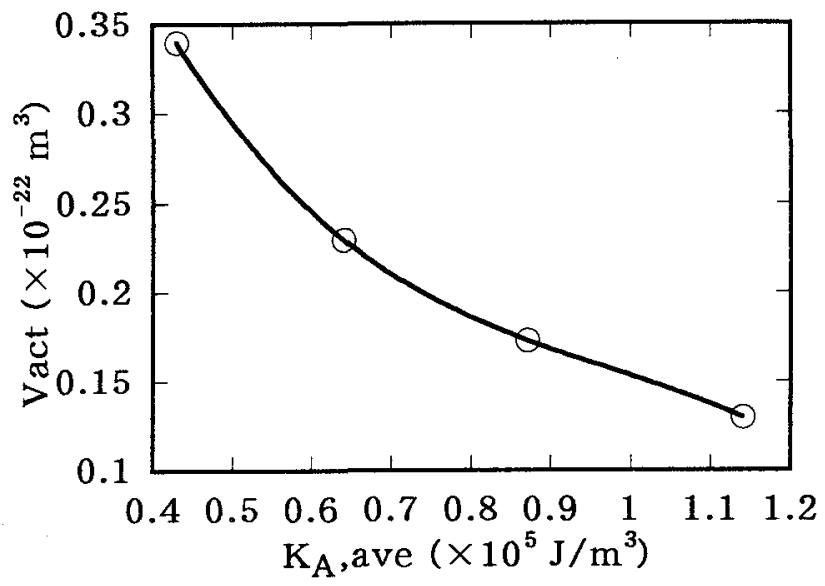

Fig. 5 Plots of the activation volume Vact against the average anisotropy constant $K_{A}$, ave for acicular Ba-ferrite particles.

Fig. 4 shows the $\mathrm{H}_{\mathrm{A}}$ distribution of acicular Ba-ferrite particles. Comparing the $\mathrm{H}_{\mathrm{A}}$ distribution of $\mathrm{X}=0$ with that of $\mathrm{X}=0.5-1.0$, the $\Delta \mathrm{Ji} / \mathrm{Js}$ versus $\mathrm{H}$ curve of $\mathrm{X}=0$ is more sharply peaked. In acicular Ba-ferrite particles, $\mathrm{H}_{\mathrm{A}}$,ave increases with increasing $\mathrm{Hcj}$ and the value of Vact is inversely proportional to $\mathrm{K}_{\mathrm{A}}$, ave, which was calculated using the value of $H_{A}$, ave as shown in Fig. 5.

\section{CONCLUSIONS}

This paper reports on the dependence of Sv, Vact, $\mathrm{Rh}$ and $\mathrm{H}_{\mathrm{A}}$, ave on Hcj for acicular Ba-ferrite particles prepared by a conventional sintering method. The results are summarized as follows:

1) Hcj is proportional to Vact ${ }^{2.2}$, as in the case of the platelet Ba-ferrite and SrM particles(used in magnets), and $\mathrm{SrW}$ and $\mathrm{Sm}_{2} \mathrm{Fe}_{17} \mathrm{~N}_{\mathrm{x}}$ magnets. They all have a similar mechanism of magnetization reversal:

2) $H_{A}$,ave increases with increasing Hcj and Vact is inversely proportional to $K_{A}$, ave. Rh and Vact both decrease, as Hcj increases, to approach the coherent rotation mode.

\section{ACKNOWLEDGMENT}

The author would like to thank Dr. S. Saito, excutive managing director, Dr. T. Nomura, general manager of materials research center and Mr. T. Ochiai, adviser both of TDK Corp. for their continued encouragement.

\section{REFERENCES}

[1] O. Kubo, T. Ido and H. Yokoyama, IEEE Trans. Magn., vol.23 [5], pp.3140-3142, 1987.

[2] D. E. Speliotis, J.Appl. Phys., vol.63 [8], pp.3432$3434,1988$.

[3] A. R. Corradi, D. E. Speliotis, G. Bottoni, D. Candolfo, A. Cecchetti and F. Masoli, IEEE Trans. Magn., vol.25 [5], pp.4066 -4068, 1989.

[4] H. Nishio, J.Magn. Soc. Jpn., vol. 18 [S1], pp.249252, 1994.

[5] M. Fujita, K. Kakizaki, N. Hiratsuka and K. Haneda, Proc. 6th Int. Conf. on Ferrite (Tokyo, 1992), pp.968-971.

[6] K. Kakizaki, N. Hiratsuka and T. Namikawa, J.Magn. Soc. Jpn., vol. 19 [S2], pp.67-70, 1995.

[7] H. Nishio, J.Magn. Soc. Jpn., vol.13 [S1], pp.697$702,1989$.

[8] R. Street and J. C. Woolly, Proc. Phys. Soc., vol:A62, pp.562-572, 1949.

[9] E. P. Wohlfarth, J.Phys., vol.F14, pp.L155-L159, 1984.

[10] P. Gaunt, J.Appl. Phys., vol.59 [12], pp.41294132, 1986.

[11] I. S. Jacobs and F. E. Luborsky, J.Appl. Phys., vol.28 [4], pp.467-473, 1957.

[12] S. Kitahata and M. Kishimoto, J.Magn. Soc. Jpn., vol.15 [S2], pp.693-696, 1991.

[13] J. C.Barbier, Ann. Phys. (Paris), vol.9, pp.84-141, 1954.

[14] H. Nishio, H. Taguchi, F. Hirata and T. Takeishi, IEEE Trans. Magn., vol.29 [6], pp.2637-2639, 1993.

[15] H. Nishio and H. Yamamoto, J. Phys. N France, vol. 7, pp.C1-317-381, 1997.

[16] H. Nishio, C. Ishizaka, T. Hidaka, A. Fukuno and T. Yoneyama, Proc. 7th Int. Symp. on Magnetic Anisotropy and Coercivity in RE.TM Alloy (Birmingham, 1994), pp.423-435. 